\title{
Putting the Irrelevance Back Into the Problem of Irrelevant Conjunction*
}

\author{
Branden Fitelson $†$ \\ Department of Philosophy \\ San José State University
}

\begin{abstract}
Naive deductive accounts of confirmation have the undesirable consequence that if $E$ confirms $H$, then $E$ also confirms the conjunction $H \& X$, for any $X$-even if $X$ is utterly irrelevant to $H$ (and $E$ ). Bayesian accounts of confirmation also have this property (in the case of deductive evidence). Several Bayesians have attempted to soften the impact of this fact by arguing that - according to Bayesian accounts of confirmation- $E$ will confirm the conjunction $H \& X$ less strongly than $E$ confirms $H$ (again, in the case of deductive evidence). I argue that existing Bayesian "resolutions" of this problem are inadequate in several important respects. In the end, I suggest a new-and-improved Bayesian account (and understanding) of the problem of irrelevant conjunction.
\end{abstract}

1. Introduction. The problem of irrelevant conjunction (a.k.a., the tacking problem) was originally raised as a problem for naive hypotheticodeductive (H-D) accounts of confirmation. ${ }^{1}$ According to the (naive) $\mathrm{H}-$ D account of confirmation, $E$ confirms $H$ relative to background $K$ (roughly) if $H \& K$ (classically) deductively entails $E$ (i.e., if $H \& K \vDash$ $E$ ). Therefore, owing to the monotonicity of $\vDash$, we have the following fact about (naive) $\mathrm{H}-\mathrm{D}$-confirmation:

*Received May 2002, revised July 2002.

$\dagger$ Send reprint requests to the author, Department of Philosophy, San José State University, One Washington Square, San José, CA 95192-0096.

¥Thanks to Ellery Eells, Malcolm Forster, Ken Harris, Patrick Maher, Elliott Sober, and an anonymous referee of this journal for useful comments and suggestions.

1. See Hempel 1945 for the original (classic) presentation of H-D confirmation, and some of its shortcomings (including the problem of irrelevant conjunction). See Skyrms 1992 for an incisive and illuminating critical survey of some more recent papers on deductive accounts of confirmation and the problem of irrelevant conjunction. And, see Earman 1992, 63-65 for a canonical contemporary Bayesian discussion of the problem of irrelevant conjunction.

Philosophy of Science, 69 (December 2002) pp. 611-622. 0031-8242/2002/6904-0007\$10.00

Copyright 2002 by the Philosophy of Science Association. All rights reserved. 
If $E$ H-D confirms $H$ relative to $K$,

then $E$ also H-D-confirms $H \& X$ relative to $K$, for any $X$.

The problem with (1) is supposed to be that conjoining $X$ 's that are utterly irrelevant to $H$ and $E$ seems (intuitively) to undermine the confirmation $E$ provides for the resulting (conjunctive) hypothesis $H \& X$. For instance, intuitively, the return of Halley's comet in $1758(E)$ confirmed Newton's theory $(H)$ of universal gravitation (relative to the background evidence $(K)$ available at the time). But, according to the H-D account of confirmation, this implies that the return of Halley's comet also confirms the conjunction of $H$ and (say) Coulomb's Law (or any other proposition(s) one would like to conjoin to $H$ ). And, no matter how many irrelevancies are conjoined to $H, E$ will continue to confirm the conjunction, according to the $\mathrm{H}-\mathrm{D}$ account of confirmation.

Because probabilistic correlation is not monotonic, Bayesian (incremental) confirmation does not have the property expressed in (1). That is, according to Bayesianism, it does not follow from $E$ 's confirming $H$ that $E$ must also confirm $H \& X$, for any $X$. For contemporary Bayesians, " $E$ confirms $H$ relative to $K$ " just means that for an appropriate Bayesian probability function $\operatorname{Pr}$ (for an agent), $\operatorname{Pr}(H \mid E \& K)>\operatorname{Pr}(H \mid K)$. And, in lots of cases, evidence $E$ may confirm a hypothesis $H$ in this probabilistic way without confirming some conjunction $H \& X$-i.e., without also making $\operatorname{Pr}(H \& X \mid E \& K)>\operatorname{Pr}(H \& X \mid K)$. So, Bayesianism is immune from the original problem of irrelevant conjunction. However, Bayesian confirmation does still suffer from this problem in the case of deductive evidence. That is, Bayesian confirmation and H-D confirmation both satisfy the following special case of (1), provided that $E$ is less than certain on $K-$ i.e., provided that $\operatorname{Pr}(E \mid K)<1$. $^{2}$

If $H \& K \vDash E$, then $E$ confirms $H \& X$ relative to $K$, for any $X$.

That is, when $H \& K \vDash E$ we have, for any $X, \operatorname{Pr}(E \mid H \& X \& K)=1$; thus $\operatorname{Pr}(H \& X \mid E \& K)=\operatorname{Pr}(E \mid H \& X \& K) \cdot \operatorname{Pr}(H \& X \mid K) / \operatorname{Pr}(E \mid K)>$ $\operatorname{Pr}(H \& X \mid K)$, provided that $\operatorname{Pr}(E \mid K)<1$. This is the traditional way of framing the problem of irrelevant conjunction.

2. Existing Bayesian Resolutions of the Traditional Problem. Several Bayesians have tried to resolve the traditional problem of irrelevant conjunc-

2. It is well-known that evidence which is known with certainty is powerless to confirm any hypothesis, on a (personalistic) Bayesian relevance account of confirmation (see Glymour 1980, 63-69 for the original statement of this fact, which is now infamously known as "the problem of old evidence"). Hereafter, I will assume without comment that $\operatorname{Pr}(E \mid K)<1$. 
tion by proving various quantitative results about the degree to which $E$ confirms $H$ versus $H \& X$ (relative to $K$ ) in the case of deductive evidence. One obvious Bayesian move is to point out that for any $X, \operatorname{Pr}(H \mid E \& K)$ $\geq \operatorname{Pr}(H \& X \mid E \& K)$, and that given $H \& K \vDash E$, equality holds in the special case where $\operatorname{Pr}(X \mid H \& K)=1$. So, in the deductive case $H$ is always made at least as probable as $H \& X$ by $E$ (relative to $K$ ), and in most cases is made more probable.

Although this may appear to solve the problem of irrelevant conjunction, the solution turns on the trivial fact that conjunctions are never more probable than their conjuncts. And it turns on reading the "confirms more" relation as the "makes more probable than" relation. But this is not a good interpretation of the "confirms more" relation. For, on this reading, when we compare the confirmation of hypotheses by evidence in more general cases it will often happen that " $E$ confirms $H_{2}$ more strongly than $E$ confirms $H_{1}$ (relative to $K$ )" (because $\operatorname{Pr}\left(H_{2} \mid E \& K\right)>\operatorname{Pr}\left(H_{1} \mid E \&\right.$ $K)$ ), while $E$ actually lowers the probability of $H_{2}\left(\operatorname{Pr}\left(H_{2} \mid E \& K\right)<\right.$ $\left.\operatorname{Pr}\left(H_{2} \mid K\right)\right)$ and raises the probability of $H_{1}\left(\operatorname{Pr}\left(H_{1} \mid E \& K\right)>\operatorname{Pr}\left(H_{1} \mid K\right)\right)$. So, the brute comparison of the relative sizes of posterior probabilities is intuitively an unappealing way to cash out the "confirms more" relation (see Popper 1954 for an early and forceful argument for this claim).

How then are we to cash out "confirms more"? Clearly, we want to employ some measure of how much the posterior probability of $H$ is raised by $E$ over its prior probability. I will call such measures relevance measures. Several relevance measures of confirmation have occurred to Bayesians (see Kyburg 1983, Fitelson 1999, and Festa 1999 for surveys). The most common are the ratio measure, the difference measure, and the likelihoodratio measure. The ratio measure would measure the amount by which $E$ confirms $H$ (relative to $K$ ) as the ratio $r(H, E \mid K)=\operatorname{Pr}(H \mid E \& K) / \operatorname{Pr}(H \mid K)$. This measure would have it that $E$ confirms $H_{2}$ more strongly than $H_{1}$ (relative to $K)$ just when the ratio $r\left(H_{2}, E \mid K\right)$ is larger than the ratio $r\left(H_{1}\right.$, $E \mid K)$. The difference measure gauges the degree to which $E$ confirms $H$ (relative to $K)$ in terms of the difference $d(H, E \mid K)=\operatorname{Pr}(H \mid E \& K)-$ $\operatorname{Pr}(H \mid K)$. On this measure, $E$ confirms $H_{2}$ more strongly than $H_{1}$ (relative to $K)$ just when the difference $d\left(H_{2}, E \mid K\right)$ is larger than the difference $d\left(H_{1}, E \mid K\right)$. Finally, according to the likelihood-ratio measure, the degree to which $E$ confirms $H$ (relative to $K$ ) is given by the likelihood ratio $l(H$, $E \mid K)=\operatorname{Pr}(E \mid H \& K) / \operatorname{Pr}(E \mid \bar{H} \& K){ }^{3}$ And, according to $l, E$ confirms $H_{2}$

3. I am using overbars to indicate (classical) logical negations of propostions (e.g., ' $\bar{X}$ ' is to be read 'not $\left.X^{\prime}\right)$. It is easy to show that $l(H, E \mid K)=[\operatorname{Pr}(H \mid E \& K) \cdot(1-$ $\operatorname{Pr}(H \mid K))] /[(1-\operatorname{Pr}(H \mid E \& K)) \cdot \operatorname{Pr}(H \mid K)]$. So, like $d$ and $r$, the likelihood ratio measure $l$ is a function solely of the posterior and prior of $H$, and $l$ can be thought of as a measure of "the degree to which $E$ raises the probability of $H$ (relative to $K$ )". It may 
more strongly than $E$ confirms $H_{1}$ just in case the ratio $l\left(H_{2}, E \mid K\right)$ is larger than the ratio $l\left(H_{1}, E \mid K\right)$. There is much controversy in the literature as to which of these three measures (or some other measure altogether ${ }^{4}$ ) is the "one true (relevance) measure of (incremental) confirmation". No clear consensus has been reached amongst Bayesians (see Fitelson 2001 for an extended discussion of this controversy and some attempts to resolve it). Until such a consensus is reached, the best policy is to look for highly robust solutions to problems involving these measures - solutions that hold (simultaneously) for as many of the competing relevance measures as possible.

With regard to the problem of irrelevant conjunction, Earman's analysis employs the difference measure $d$. Earman (1992, 63-65) proves the following:

$$
\text { If } H \& K \vDash E \text {, then } d(H \& X, E \mid K)<d(H, E \mid K) .
$$

In other words, Earman argues that, while $E$ does (from a Bayesian point of view) continue to confirm $H \& X$ in the case of deductive evidence, the degree to which $E$ confirms $H \& X$ will be less than the degree to which $E$ confirms $H$ (relative to background evidence $K$ ). And, as more and more irrelevant conjuncts are added, the degree to which $E$ confirms the conjunction will tend to decrease. Earman's Bayesian approach to this problem is sensitive to choice of measure of confirmation - sensitive to precisely which function $r, d, l$, or some other function, is employed to represent the amount by which $E$ confirms $H$ (relative to $K$ ). That is, in Fitelson 1999 and Fitelson 2001, it is shown that (2) is not true if the difference measure $d$ is replaced by the ratio $r$ of the posterior probability of $H$ (on $E$ ) to the prior probability of $H$ (relative to $K$ ). ${ }^{5}$ But, because Earman's (2) holds for all other Bayesian relevance measures that have been proposed, his argument can be inoculated against the measure sensitivity problem simply by providing compelling reasons to reject the ratio mea-

also help to think of the likelihood ratio $l$ as the ratio of the posterior to the prior odds of $H$ (given $K$ ). See Good 1985 for a nice discussion concerning the likelihood-ratio measure and its rather illustrious history.

4. There are many other candidates in the literature. Recently, Joyce (1999) and Christensen (1999) have defended the measure $s(H, E \mid K)=\operatorname{Pr}(H \mid E \& K)-\operatorname{Pr}(H \mid \bar{E} \& K)$. Some of the salient properties of $s$ are discussed in detail in Eells and Fitelson 2000 and Fitelson 2001.

5. The ratio measure of degree of confirmation has been used and defended by many philosophers of science (see, e.g., Milne 1996). Earman (1992) has an argument of his own against the ratio measure, but (as Milne (1996) points out) this argument begs the question in the context of the problem of irrelevant conjunction. In Fitelson 2001 and Eells and Fitelson 2002, several compelling arguments against the ratio measure are presented and discussed. 
sure of degree of confirmation (see Fitelson 2001 and Eells and Fitelson 2002 for several such reasons). However, it seems to me that Earman's analysis of irrelevant conjunction has other, more telling shortcomings.

A closer look at (2) reveals that the irrelevance of $X$ is absent from the Bayesian analysis and resolution of the problem of irrelevant conjunction. What (2) says is that, when $H \& K \vDash E$, as conjuncts $X$ (simpliciter) are added to $H$, the degree to which $E$ confirms $H \& X$ will tend to decrease. As far as (2) is concerned, a conjunct $X$ could be (intuitively) relevant to $H$ and $E$, but this would not prevent the conjunction $H \& X$ from being less strongly confirmed than $H$ by $E$. This is unfortunate, for two reasons. First, it was supposed to be the irrelevant $X$ 's that made (1) and (1') seem so unattractive. It's not so obvious that either (1) or $\left(1^{\prime}\right)$ is incorrect in the case of highly positively relevant $X$ 's. Moreover, Bayesian confirmation theory is founded on a perfectly precise and intuitive kind of relevance (viz., correlation), which is not mentioned anywhere in the "Bayesian" statements or resolutions of the problem of irrelevant conjunction. So, Bayesians who endorse Earman's resolution (grounded in (2)) have apparently both $(i)$ lost track of which $X$ 's were supposed to make (1) and $\left(1^{\prime}\right)$ seem so unintuitive; and, in the process, (ii) forsaken the very notion of relevance that undergirds their own theory of confirmation.

Rosenkrantz (1994) does seem somewhat sensitive to these points. Rosenkrantz motivates his (alternative) Bayesian resolution of the problem of irrelevant conjunction, as follows. ${ }^{6}$

On $\mathrm{H}-\mathrm{D}$ accounts, $H$ is confirmed by a verified prediction, $E$, but $E$ is equally a prediction of $H \& X$, where the 'tacked on' $X$ may be a quite extraneous hypothesis. ... There are those who think that this sin of 'irrelevant conjunction' vitiates Bayesian confirmation theory as well. ... I hope you will agree that the two extreme positions on this issue are equally unpalatable, (i) that a consequence $E$ of $H$ confirms $H$ not at all, and (ii) that $E$ confirms $H \& X$ just as strongly as it confirms $H$ alone. . . In general, intuition expects intermediate degrees of confirmation that depend on the degree of compatibility of $H$ with $X$. Measuring degree of confirmation by $[d(H, E \mid K)=$ $\left.{ }_{d f} \operatorname{Pr}(H \mid E \& K)-\operatorname{Pr}(H \mid K)\right] \ldots$ yields $[d(H \& X, E \mid K)=\operatorname{Pr}(X \mid H \&$ $K) \cdot d(H, E \mid K)$, in the case of deductive evidence]. (Rosenkrantz 1994, 470-471)

In other words, Rosenkrantz suggests that we should measure the degree to which $E$ confirms $H$ (relative to $K$ ) by taking the difference $(d)$ between the posterior probability of $H$ (on $E$ ) and the prior probability of $H$ (rela-

6. I have translated Rosenkrantz's (1994, 470-471) passage into my notation and added bracketed summary remarks in my parlance. 
tive to $K$ ). Then, Rosenkrantz bases his alternative Bayesian resolution of the problem of irrelevant conjunction on the following theorem (which is really just a way of precisely filling out Earman's (2)):

If $H \& K \vDash E$, then $d(H \& X, E \mid K)=\operatorname{Pr}(X \mid H \& K) \cdot d(H, E \mid K)$.

Rosenkrantz deserves credit here for trying to bring the irrelevance back into the Bayesian resolution of this problem. However, his account has two serious flaws. First, it is shown in Fitelson 1999 and Fitelson 2001 that Rosenkrantz's account is even more sensitive to choice of relevance measure of confirmation than Earman's (2)-based account. That is, if we replace $d$ with either $r$ or $l$ or any of several other currently proposed Bayesian relevance measures of the degree to which evidence confirms hypotheses, Rosenkrantz's analysis doesn't hold up. ${ }^{7}$ So, Rosenkrantz's proposal doesn't have the kind of robustness that solutions should have under the present circumstances, where we have no compelling reasons to accept $d$ as opposed to these other Bayesian relevance measures (e.g., $l$ ) of the degree to which bits of evidence confirm hypotheses.

In addition, Rosenkrantz's account makes use of a strange-and decidedly non-Bayesian - notion of "relevance." Rosenkrantz seems to be suggesting that a conjunct $X$ should be considered "irrelevant" to $H$ (relative to background $K)$ if $\operatorname{Pr}(X \mid H \& K)<1 .{ }^{8}$ This suggestion is inadequate for two reasons. First, since when do Bayesians think that the degree to which $X$ is relevant to $H$ can be measured using only the conditional probability $\operatorname{Pr}(X \mid H \& K)$ ? Secondly, the inequality $\operatorname{Pr}(X \mid H \& K)<1$ can, at best, only tell us when $X$ is "irrelevant" to $H$-it can say nothing about whether $X$ is "irrelevant" to $E$, or to various logical combinations of $H$ and $E$. It seems to me that the cases in which (1) and $\left(1^{\prime}\right)$ are least intuitive are cases in which $X$ is (intuitively) irrelevant to both $H$ and $E$, and to all logical combinations of $H$ and $E$. Indeed, it seems to me that an adequate Bayesian solution to the problem of irrelevant conjunction should be directly extendable to non-deductive cases, and should draw on a probabilistic notion of the relevance of $X$. The deductive case should turn out to be just

7. In particular, in Fitelson 1999 it is shown that Rosenkrantz's argument (unlike Earman's) fails to go through if one adopts the likelihood ratio measure $l$ of degree of confirmation. Unfortunately, Rosenkrantz (1981, Exercise 3.6) admits that he knows of "no compelling considerations that adjudicate between" the difference measure $d$ and the likelihood-ratio measure $l$. This puts Rosenkrantz in a rather uncomfortable position regarding his proposed resolution of the problem of irrelevant conjunction. In Fitelson 2001, more general facts about the measure sensitivity of Rosenkrantz's argument are established.

8. This is the (necessary and sufficient) condition under which Rosenkrantz's (3) entails a decrease in the degree of $d$-confirmation $E$ provides for $H \& X$ versus $H$ (relative to $K$ ). 
a special case of a more general rule governing confirmationally irrelevant conjunctions. Thus, I suggest that we need a new understanding of the problem of irrelevant conjunction, as seen from a Bayesian perspective.

3. A New Bayesian Perspective and Resolution. I suggest that we go about this in an entirely different way. Let's start by saying what it means (in a Bayesian framework) to say that $X$ is confirmationally irrelevant to $H, E$, and all logical combinations of $H$ and $E$. Then, once we have this precisely defined, let's see if (and under what auxiliary assumptions) we can show that such irrelevant conjuncts lead to decreased confirmational power. The first of these tasks is already done. Bayesians already have a perfectly precise definition of confirmational irrelevance: probabilistic independence. So, let's say that $A$ is confirmationally irrelevant to $B$ (relative to $K$ ) just when $A$ and $B$ are probabilistically independent (given $K$ )-i.e., when $\operatorname{Pr}(A \& B \mid K)=\operatorname{Pr}(A \mid K) \cdot \operatorname{Pr}(B \mid K)$. Therefore, we already know what it means (in a Bayesian confirmation-theoretic framework) to say that $X$ is confirmationally irrelevant to $H, E$, and all logical combinations $H$ and $E$. The next step is to note that Bayesian confirmation theory does fall prey to its own general, qualitative problem of irrelevant conjunction. That is, we have the following theorem (hereafter, "confirms" is used in the Bayesian, relevance sense - see the Appendix for proofs of theorems):

Theorem 1. If $E$ confirms $H$, and $X$ is confirmationally irrelevant to $H, E$, and $H \& E$ (relative to $K$ ), then $E$ also confirms $H \& X$ (relative to $K$ ).

I claim that this is the "problem of irrelevant conjunction" that Bayesian confirmation theorists should be worrying about. The good news is that we can prove the following quantitative theorem, which seems to be the relevant result for Bayesian confirmation theory and the problem of irrelevant conjunction:

Theorem 2. If $E$ confirms $H$, and $X$ is confirmationally irrelevant to $H, E$, and $H \& E$ (relative to $K$ ), then (provided that $\operatorname{Pr}(X \mid K)<1^{9}$ ):

$$
\mathfrak{c}(H, E \mid K)>\mathfrak{c}(H \& X, E \mid K),
$$

where $\mathrm{c}$ may be either the difference measure $d$ or the likelihood-ratio measure l of degree of confirmation (but, $c$ may not be the ratio measure $r$, since in cases of irrelevant conjunction we will have $r(H, E \mid K)=r(H \& X, E \mid K))$.

9. If $\operatorname{Pr}(X \mid K)=1$, then $X$ can hardly be thought of as a conjunct, since in such cases $H$ and $H \& X$ will be probabilistically indistinguishable (relative to $K$ ). So, since we are presently interested in the problem of irrelevant conjunction, it seems quite natural to assume that $\operatorname{Pr}(X \mid K)<1$. 
Our Bayesian analysis and resolution of the problem of irrelevant conjunction has the following advantages over its existing rivals:

- Our analysis makes use of the irrelevance of $X$. Moreover, our notion of confirmational irrelevance is not some peculiar one (like Rosenkrantz's), but just the standard Bayesian notion of probabilistic independence.

- Our resolution is not restricted to the (not very inductively interesting) special case of deductive evidence; it explains why irrelevant conjuncts are confirmationally disadvantageous in all contexts (deductive or otherwise).

- Our resolution is as robust (i.e., measure-insensitive) as any other existing resolution (e.g., Earman's), and more robust than any other existing account that tries to be sensitive to the "irrelevance" of the conjunct $X$ (e.g., Rosenkrantz's). That is, our account is based on a theorem which holds for all Bayesian measures of confirmation currently being used in the philosophical literature, except for the ratio measure $r .^{10}$

\section{Appendix: Proofs of Theorems}

\section{A Proof of Theorem 1}

Theorem 1. If $E$ confirms $H$, and $X$ is confirmationally irrelevant to $H$, $E$, and $H \& E$ (relative to $K$ ), then $E$ also confirms $H \& X$ (relative to $K$ ).

Proof. The proof of this theorem is easy. ${ }^{11}$ We assume without loss of generality that $K$ is tautologous, and we reason as follows:

10. In Fitelson 2001, it is shown that our Theorem 2 holds for all Bayesian measures of degree of confirmation currently used or defended in the philosophical literature, except for the ratio measure $r$. Apparently, $r$ cannot be used to resolve the problem of irrelevant conjunction - even in cases that do not involve deductive evidence. This shows an even deeper problem with $r$ than the (mere) "deductive insensitivity," which prevents $r$ from satisfying Earman's (2) (see Fitelson 2001, 2.2 .2 .1 and $\$ 2.3 .1$ for further discussion). Defenders of $r$ (e.g., Milne (1996)) are quick to point out that $r$ 's (mis) handling of the traditional, deductive problem of irrelevant conjunction is not such a serious weakness of $r$. Indeed, Milne (1996) characterizes the traditional problem of irrelevant conjunction as a "wretched shibboleth" in this context. I am somewhat sympathetic to this point of view. Deductive cases are not terribly interesting from an inductive-logical point of view. However, I think that $r^{6}$ s mishandling of irrelevant conjuncts in the inductive case (as in our Theorem 2) ought to be taken more seriously. See Fitelson 2001 for further problems with the ratio measure $r$, and a critical analysis of Milne's (1996) desideratum/explicatum argument in favor of $r$.

11. In the Appendix we will assume that all relevant conditional probabilities are de- 


$$
\begin{aligned}
\operatorname{Pr}(H \& X \mid E) & =\frac{\operatorname{Pr}((H \& E) \& X)}{\operatorname{Pr}(E)} \quad[\text { def. of } \operatorname{Pr}(\cdot \cdot)] \\
& =\frac{\operatorname{Pr}(H \& E) \cdot \operatorname{Pr}(X)}{\operatorname{Pr}(E)} \quad[\text { irrelevance of } X] \\
& =\operatorname{Pr}(H \mid E) \cdot \operatorname{Pr}(X) \quad[\text { def. of } \operatorname{Pr}(\cdot \cdot)] \\
& >\operatorname{Pr}(H) \cdot \operatorname{Pr}(X) \quad[E \text { confirms } H] \\
& >\operatorname{Pr}(H \& X) \quad[\text { irrelevance of } X]
\end{aligned}
$$

Therefore, in the case of irrelevant conjunct $X$ and confirmatory evidence $E, \operatorname{Pr}(H \& X \mid E)>\operatorname{Pr}(H \& X)$, which completes the proof.

\section{B Proof of Theorem 2}

Theorem 2. If $E$ confirms $H$, and $X$ is confirmationally irrelevant to $H$, $E$, and $H \& E$ (relative to $K$ ), then (provided that $\operatorname{Pr}(X \mid K)<1$ ):

$$
\mathfrak{c}(H, E \mid K)>\mathfrak{c}(H \& X, E \mid K),
$$

where $\mathrm{c}$ may be either the difference measure $d$ or the likelihood-ratio measure l of degree of confirmation (but, $\mathrm{c}$ may not be the ratio measure $r$, since in cases of irrelevant conjunction we will have $r(H, E \mid K)=r(H \& X, E \mid K))$.

Proof. For the $\mathfrak{c}=d$ case of the theorem (indeed, for all three cases), we assume without loss of generality that $K$ is tautologous, and we reason as follows:

$$
\begin{aligned}
d(H \& X, E) & =\operatorname{Pr}(H \& X \mid E)-\operatorname{Pr}(H \& X) \quad \text { [def. of } d] \\
& =\frac{\operatorname{Pr}((H \& E) \& X)}{\operatorname{Pr}(E)}-\operatorname{Pr}(H \& X) \quad[\text { def. of } \operatorname{Pr}(\cdot \mid \cdot)] \\
& \left.=\frac{\operatorname{Pr}(H \& E) \cdot \operatorname{Pr}(X)}{\operatorname{Pr}(E)}-\operatorname{Pr}(H) \cdot \operatorname{Pr}(X) \quad \text { [irrelevance of } X\right] \\
& =\operatorname{Pr}(X) \cdot[\operatorname{Pr}(H \mid E)-\operatorname{Pr}(H)] \quad[\text { def. of } \operatorname{Pr}(\cdot \cdot), \text { algebra }] \\
& =\operatorname{Pr}(X) \cdot d(H, E) \quad[\text { def. of } d] \\
& <d(H, E) \quad[\operatorname{Pr}(X)<1, d(H, E)>0]
\end{aligned}
$$

fined. In fact, we may assume that all probabilities involved are non-extreme (this leads to no real loss of generality, since what we're talking about are inductive cases of irrelevant conjunction). 
For the $\mathrm{c}=r$ case of the theorem, we will prove that $r(H, E)=r(H \&$ $X, E$ ), when $X$ is an irrelevant conjunct (hence, that $r$ violates the theorem).

$$
\begin{aligned}
r(H \& X, E) & \left.=\frac{\operatorname{Pr}(H \& X \mid E)}{\operatorname{Pr}(H \& X)} \quad \text { [def. of } r\right] \\
& \left.=\frac{\operatorname{Pr}((H \& E) \& X)}{\operatorname{Pr}(E) \cdot \operatorname{Pr}(H \& X)} \quad \text { [def. of } \operatorname{Pr}(\cdot \cdot)\right] \\
& \left.=\frac{\operatorname{Pr}(H \& E) \cdot \operatorname{Pr}(X)}{\operatorname{Pr}(E) \cdot \operatorname{Pr}(H) \cdot \operatorname{Pr}(X)} \quad \text { [irrelevance of } X\right] \\
& =\frac{\operatorname{Pr}(H \mid E)}{\operatorname{Pr}(H) \quad[\text { def. of } \operatorname{Pr}(\cdot \cdot), \text { algebra] }} \\
& =r(H, E) \quad \text { [def. of } r]
\end{aligned}
$$

We are now ready to prove the likelihood-ratio $(l)$ case of the theorem (via reductio ad absurdum, and using the $d$ and $r$ results as lemmas).

$$
\begin{array}{ll}
l(H \& X, E) \geq l(H, E) & \text { [reductio assumption] } \\
\frac{\operatorname{Pr}(E \mid H \& X)}{\operatorname{Pr}(E \mid \overline{H \& X})} \geq \frac{\operatorname{Pr}(E \mid H)}{\operatorname{Pr}(E \mid \bar{H})} & \text { [def. of } l] \\
\frac{\operatorname{Pr}(H \& X \mid E)}{\operatorname{Pr}(H \& X)} \cdot \frac{\operatorname{Pr}(\overline{H \& X})}{\operatorname{Pr}(\overline{H \& X} \mid E)} & \\
\geq \frac{\operatorname{Pr}(H \mid E)}{\operatorname{Pr}(H)} \cdot \frac{\operatorname{Pr}(\bar{H})}{\operatorname{Pr}(\bar{H} \mid E)} & \text { [Bayes'Theorem, algebra] } \\
r(H \& X, E) \cdot \frac{\operatorname{Pr}(\overline{H \& X})}{\operatorname{Pr}(\overline{H \& X} \mid E)} & \\
\geq r(H, E) \cdot \frac{\operatorname{Pr}(\bar{H})}{\operatorname{Pr}(\bar{H} \mid E)} & {[\text { def. of } r]} \\
\frac{\operatorname{Pr}(\overline{H \& X})}{\operatorname{Pr}(\overline{H \& X} \mid E)} \geq \frac{\operatorname{Pr}(\bar{H})}{\operatorname{Pr}(\bar{H} \mid E)} & \\
(1-\operatorname{Pr}(H \mid E)) \cdot(1-\operatorname{Pr}(H \& X)) \geq & {[\operatorname{Pr}(\bar{X} \mid Y)=1-\operatorname{Pr}(X \mid Y), \text { algebra] }}
\end{array}
$$




$$
\begin{aligned}
& {[d(H \& X, E)-d(H, E)]+\operatorname{Pr}(H \mid E) \cdot \operatorname{Pr}(H \& X)} \\
& \quad \geq \operatorname{Pr}(H) \cdot \operatorname{Pr}(H \& X \mid E) \quad \text { [def. of } d, \text { algebra] }
\end{aligned}
$$

$$
\begin{aligned}
& \operatorname{Pr}(H \mid E) \cdot \operatorname{Pr}(H \& X) \\
& \quad>\operatorname{Pr}(H) \cdot \operatorname{Pr}(H \& X \mid E)
\end{aligned}
$$$$
r(H, E)>r(H \& X, E)
$$

$l(H \& X, E)<l(H, E)$
$[d(H \& X, E)<d(H, E)$, algebra $]$

[def. of $r$, algebra]

$[r(H \& X, E)=r(H, E)$, reductio $]$

This completes the proof of Theorem 2, as well as the Appendix. ${ }^{12}$

\section{REFERENCES}

Christensen, David (1999), "Measuring Confirmation", Journal of Philosophy 96: 437-461.

Earman, John (1992), Bayes or Bust: A Critical Examination of Bayesian Confirmation Theory. Cambridge, MA: MIT Press.

Eells, Ellery and Branden Fitelson (2000), "Measuring Confirmation and Evidence", Journal of Philosophy 97: 663-672.

(2002), "Symmetries and Asymmetries in Evidential Support", Philosophical Studies 107: $129-142$.

Festa, Roberto (1999), "Bayesian Confirmation" in Maria Carla Galavotti and Alessandro Pagnini (eds.) Experience, Reality, and Scientific Explanation. Dordrecht: Kluwer Academic Publishers, 55-87.

Fitelson, Branden (1999), "The Plurality of Bayesian Measures of Confirmation and the Problem of Measure Sensitivity", Philosophy of Science 66 (Proceedings), S362-S378. (2001), Studies in Bayesian Confirmation Theory. Ph.D. Dissertation. Madison, Wisconsin: University of Wisconsin-Madison (Philosophy). The Dissertation can be downloaded in its entirety from http://fitelson.org/thesis.pdf.

Glymour, Clark (1980), Theory and Evidence. Cambridge, MA: MIT Press.

Good, Irving John (1985), "Weight of Evidence: A Brief Survey", in Bernardo, José, Morris DeGroot, Dennis Lindley and Adrian Smith (eds.) Bayesian Statistics, 2. Amsterdam: North-Holland, 249-269.

Hempel, Carl (1945), "Studies in the Logic of Confirmation", Mind 54: 1-26, 97-121.

Joyce, James (1999), The Foundations of Causal Decision Theory. Cambridge: Cambridge University Press.

Kyburg, Henry (1983), "Recent Work in Inductive Logic", in Tibor Machan and Kenneth Lucey (eds.) Recent Work in Philosophy. Lanham: Rowman \& Allanheld, 87-150.

Milne, Peter (1996), " $\log [p(h / e b) / p(h / b)]$ is the One True Measure of Confirmation", Philosophy of Science 63:21-26.

Popper, Karl (1954), "Degree of Confirmation", The British Journal for the Philosophy of Science 5: 143-149.

Rosenkrantz, Roger (1981), Foundations and Applications of Inductive Probability. Atascadero, CA: Ridgeview.

12. See Fitelson 2001, $\$ 2.2 .2 .1$ for a more general theorem, which covers several additional measures of confirmation that have been used in the literature, including the measure $s$ (see footnote 4) recently used and defended by Christensen (1999) and Joyce (1999). 
(1994), "Bayesian confirmation: Paradise regained", The British Journal for the Philosophy of Science 45: 467-476.

Skyrms, Brian (1992), Review of "Hypothetico-Deductivism is Hopeless" by Clark Glymour, "Relevance Logic Brings Hope for Hypothetico-Deductivism" by C. Kenneth Waters, and "Truth, Content and Hypothetico-Deductive Method" by Thomas Grimes, Journal of Symbolic Logic 57: 756-758. 